\title{
Minimum Data Set for Mass-Gatherings Health Research and Evaluation: A Response
}

\author{
Sheila A. Turris, RN, PhD; Nurse Practitioner (Family); ${ }^{1}$ \\ Adam Lund, BSc, MD, MDE, FRCPC (Emergency) ${ }^{2}$
}

1. Schools of Nursing, University of British Columbia, Vancouver, British Columbia, Canada and University of Victoria, Victoria, British Columbia, Canada

2. Department of Emergency Medicine, University of British Columbia, Vancouver, British Columbia, Canada

\author{
Correspondence: \\ Sheila A. Turris, RN, PhD, Nurse \\ Practitioner (Family) \\ 6th Floor, 132 West Esplanade \\ North Vancouver V7 M 1A2 British \\ Columbia, Canada \\ E-mail: sheila@ubcmgm.ca
}

\author{
Abbreviations: \\ MDS: minimum data set \\ MG: mass gathering \\ MGH: mass-gathering health \\ RDS: required data set \\ Online publication: January 4, 2013
}

doi:10.1017/S1049023X12001707
We read the recently published manuscript entitled Minimum Data Set for MassGathering Health Research and Evaluation: A Discussion Paper with great interest. ${ }^{1}$ The authors argued that in order to advance the science of mass-gathering health (MGH), researchers and clinicians ought to employ a similar approach to data collection, using standardized conceptual definitions and uniform classifications for the illnesses and injuries that occur in the setting of mass gatherings (MGs). In essence, Ranse and Hutton argued for the creation of a "minimum data set" (MDS) or a standardized set of variables that would be of interest with regard to every mass-gathering event, regardless of country, event category, or event type.

The authors posited several key arguments that deserve serious attention within the MGH community. This paper is an important contribution to the MGH literature. After reviewing the proposed MDS requirements carefully, we have the following comments to offer. We make the comments below in the context of our work as MGH clinicians and researchers with a shared interest in the systematic collection of data across the spectrum of MGs. ${ }^{2}$

Minimum, Maximum or Required Data Sets

We appreciate that Ranse and Hutton proposed a "minimum data set" approach to data collection, including only those categories deemed essential. This approach will inevitably increase uptake by researchers and clinicians in the MGH community. However, elsewhere we have argued that a "maximum data set" for MGs provides an opportunity to capture as many potentially relevant data points as possible for different event types and categories. ${ }^{3}$ This approach may leave more room for theory building and hypothesis generation. International collaboration on a broader selection of (1) event; (2) patient; and (3) team-related factors would be of value.

We therefore propose that the authors consider renaming the MDS as the "required data set" or RDS. The goal of this change would be to expand the proposed data collection model to include optional (as opposed to required) data fields to address a wider range of variables (described below). It is our hope that the addition of optional modules will allow the proposed data set to continue to evolve over time as the science of MGH matures. We feel that Ranse and Hutton have provided a discussion piece that will unite the MGH community and that we should capitalize on this opportunity. Our goal in making the following suggestions is not to make the data collection model unwieldy, discouraging uptake by the MGH community, but rather to make it more comprehensive and adaptable to the needs of diverse researchers and clinicians.

\section{Inclusion of Optional "Modules" to Capture Additional Variables Event-Related Factors}

We wholeheartedly agree that an MDS is central to advancing our knowledge regarding the types of illnesses and injuries that occur in different types of events, across categories of events, and for all sizes of events. Ranse and Hutton argued that a focus on environmental, psychosocial, and biomedical domains, as identified by Arbon (2004), should organize and inform the selection of variables of interest within the MDS. ${ }^{4}$

- Environmental Factors Module. Given that the model underpinning the proposed MDS includes a focus on environmental factors, we urge the authors to extend the proposed MDS to include event-related factors (ie, weather conditions, crowd density, bounded vs unbounded, overnight camping, the presence and use of alcohol 
and drugs on site, etc.) thought to influence patient presentation rates and transfer to hospital rates. ${ }^{4,5}$

- Health Promotion and Illness Prevention Module. After reviewing the categories carefully, we believe that the proposed MDS would be strengthened by the inclusion of additional key variables that relate to health promotion and illness prevention. For example, variables related to mechanism of injury such as "dehydration" and "fall" would be valuable additions to the MDS. Inclusion of these variables would inform future efforts aimed at illness prevention and health promotion. It has been argued elsewhere that health promotion and illness prevention should be a focus, as this supports safer, enjoyable events. ${ }^{6}$ Including categories for health promotion and illness prevention (ie, request for athletic taping, massage therapy, sunscreen, water, etc.) in the proposed MDS should be considered as this will provide much needed data to support future research around safe, enjoyable events.

\section{Patient-Related Factors}

Along with the many valuable patient-related fields identified by Ranse and Hutton, there may be others worth considering as optional data sets:

- Acuity Determination Module. Specifically, we ask the authors to consider embedding acuity determination within the proposed MDS. Capturing data on the acuity profile of patient presentations at events will be valuable for event planners in completing risk assessments for similar events, and planning for personnel and material resource allocation. How this should be captured remains up for discussion, but using a triage scale or another measure of case mix would be worth exploring further.

- Modified ICD Codes Module. After comparing the proposed MDS to the data our team collects during events, we applaud Ranse and Hutton's focus on categorizing illness and injury based on the presenting complaint rather than the discharge diagnosis. We acknowledge that the latter is dependent on the level of health care professional on scene at a given event. We hope that as the proposed MDS evolves, it may be

References

1. Ranse J, Hutton A. Minimum data set for mass-gathering health research and evaluation: a discussion paper. Prehosp Disaster Med. 2012;27(5):1-8.

2. Mass Gathering Medicine. http://mgm.sites.olt.ubc.ca/. Accessed October 29, 2012.

3. Lund A, Turris SA, Amiri N, Lewis K, Carson M. Mass-gathering medicine: creation of an online event and patient registry. Prehosp Disaster Med. 2012;27(6): 1-11.

4. Arbon P. The development of conceptual models for mass-gathering health. Prehosp Disaster Med. 2004;19(3):208-212.

5. Milsten AM, Maguire BJ, Bissell RA, Seaman KG. Mass-gathering medical care: a review of the literature. Prehosp Disaster Med. 2002;17(3):151-162.

6. Gutman SJ, Lund A, Turris SA. Medical support for the 2009 World Police and Fire Games: a descriptive analysis of a large-scale participation event and its impact. Prehosp Disaster Med. 2009;26(1):33-40. possible to integrate an abbreviated version of International Classification of Disease (ICD) codes that would include a default category for the presenting concern if that is the best data available.

\section{On-site Health Care Team}

In addition, it has been argued that the presence of an organized health care team at a given event may reduce hospital transfers. ${ }^{5,7-11}$ Some events operate at a "first aid only" capacity, while others have a larger, interdisciplinary team. It has been suggested that the presence of physicians, nurse practitioners, and/or other clinical decision makers may decrease the need to transfer patients by ambulance.

- Team Composition Module. Capturing the composition of the on-site team in the MDS would be ideal. There would also be value in quantifying the number of team members at each level of training, and finding a way to categorize deployment (ie, number of treatment stations, shifts, variable coverage, etc.).

- Treatment Capacity Module. Based on our work at events, there would be tremendous value-from a planning and event modeling perspective-in collecting data regarding the types of treatments offered onsite during events. This type of information may shed some light on the level of care required to influence transfer to hospital rates and ambulance transfer rates within a given category of events, or for a specific event.

The issues raised by Ranse and Hutton highlight the importance of international collaboration and leadership in creating a uniform approach to data collection and analysis. The adoption and evolution of the MDS will depend on ongoing dialogue within the MGH community. The 2013 World Congress on Disaster and Emergency Medicine conference in Manchester will permit an opportunity for diverse input and perhaps the foundation for a consensus document on a MG research framework.

In summary, we feel that the proposed MDS represents a great stride forward for MG research and we applaud Ranse and Hutton for making this important contribution to MG literature.

7. Grange JT, Baumann GW, Vaezazizi R. On-site physicians reduce ambulance transports at mass gatherings. Prehosp Emerg Care. 2003;7:322-326.

8. Milsten AM, Seaman KG, Liu P, Bissell RA, Maguire BJ. Variables influencing medical usage rates, injury patterns, and levels of care for mass gatherings. Prehosp Disaster Med. 2003;18(4):334-346.

9. McDonald CC, Koenigsberg MD, Ward S. Medical control of mass gatherings: can paramedics perform without physicians on-site? Prehosp Disaster Med. 1993;8(4): 327-331.

10. Olapade-Olaopa E, Alonge TO, Amanor-Boadu SD, Alese OB, Adeyinka AL, Ogunbunmi PA. On-site physicians at a major sporting event in Nigeria. Prehosp Disaster Med. 2006;21(1):40,41-44.

11. Rubin CB, Renda-Tanali I. Effects of the terrorist attacks on Sept. 11, 2001, on federal emergency management. J of Am Soc of Prof Emerg Planners. 2002;1(1):1-18. 


\title{
Author Reply: \\ Minimum Data Set for Mass-Gatherings Health \\ Research and Evaluation: The Beginning of an \\ International Dialogue
}

\author{
Jamie Ranse, RN, FACN, FCENA, BN, GCertClinEd, GCertClinEpi, MCritCarNurs; ${ }^{1,2}$ \\ Alison Hutton, RN, Dip of Ap. Sci(Nsg), BN, MN, PhD, MACN ${ }^{2}$
}

1. Faculty of Health, University of Canberra, Canberra, Australian Capital Territory, Australia

2. School of Nursing and Midwifery, Flinders

University, Adelaide, South Australia, Australia

\section{Correspondence:}

Jamie Ranse, RN, BN, McritCarNurs

Faculty of Health

University of Canberra

Canberra, Australia, ACT, 2601

E-mail: jamie@jamieranse.com
In writing our original paper ${ }^{1}$ we aimed at generating international discussion regarding the need for international consistency in the recording and reporting of biomedical information relating to research and evaluation undertaken within the mass gathering health environment. Following a response from Turris and Lund, ${ }^{2}$ we are excited to now continue this discussion.

We would like to thank Turris and Lund for their time to consider a response ${ }^{2}$ to our original work. ${ }^{1}$ Such a reply indicates an international need to carry on the conversation regarding a collective agreed set of variables that would enhance the ability of clinicians, researchers, event managers and others, locally, nationally and internationally, to contribute to the understanding of mass gathering health.

For the remainder of this response, we add to the discussion generated by our previous work:

1. Our original work ${ }^{1}$ focused on patient-level biomedical aspects of mass gathering health. As such, we consciously decided not to include a review, discussion or recommendations related to additional domains or modules that could, and would, be required to constitute a comprehensive collection of data. To include this additional information would have resulted in a much larger manuscript, in which the key messages would have been lost. As such, our list of variables is not exhaustive and we acknowledge that many other known variables, from other domains, could be included. Therefore, we agree with Turris and Lund ${ }^{2}$ that the international conversation should be much broader than the patient-level biomedical aspects alone.

2. Turris and Lund suggest that we should consider renaming our proposed minimum data set as the "required data set." Yet, regardless of whether the information collected is within a minimum, maximum or required data set is somewhat irrelevant. The primary concept is that the information collected should be internationally relevant and standardized to allow clear interpretation and transferability of how we understand, interpret and implement mass-gathering health strategies. As a consequence the ability to provide and plan clinical services at mass-gathering events locally, nationally and internationally will be enhanced; this was an important motivator in developing our original work.

3. The need for international collaboration and leadership in mass-gathering events is increasing. We concur with Turris and Lund that the $18^{\text {th }}$ World Congress on Disaster and Emergency Medicine in Manchester will provide a foundational opportunity in building mass-gathering health leadership and we look forward to future possible international collaborations.

Overall, the response and discussion to our original work is very encouraging in building an international understanding and collaboration relating to mass gatherings from a health perspective.

References

1. Ranse J, Hutton A. Minimum data set for mass-gathering health research and evaluation: a discussion paper. Prehosp Disaster Med. 2012;27(5):1-8.

2. Turris S, Lund A. Minimum Data Set for Mass-Gatherings Health Research and Evaluation: A Response. Prehosp Disaster Med. In press. 